\title{
Colloque « Tout un monde en santé ! Enjeux pour l'éducation relative à l'environnement »
}

\section{Sandrine Louapre}

\section{OpenEdition}

1 Journals

Édition électronique

URL : https://journals.openedition.org/ere/8337

ISSN : 2561-2271

\section{Éditeur}

Centr'ERE

\section{Référence électronique}

Sandrine Louapre, "Colloque « Tout un monde en santé ! Enjeux pour l'éducation relative à

l'environnement » », Éducation relative à l'environnement [En ligne], Volume 17-1 | 2022, mis en ligne le 27 janvier 2022, consulté le 02 février 2022. URL : http://journals.openedition.org/ere/8337

\section{Ce document a été généré automatiquement le 2 février 2022}




\title{
Colloque « Tout un monde en santé ! Enjeux pour l'éducation relative à l'environnement »
}

\author{
Sandrine Louapre
}

\section{NOTE DE L'AUTEUR}

Avec la collaboration de Jacqueline Romano-Toramanian, Lucie Sauvé et Jean Robitaille.

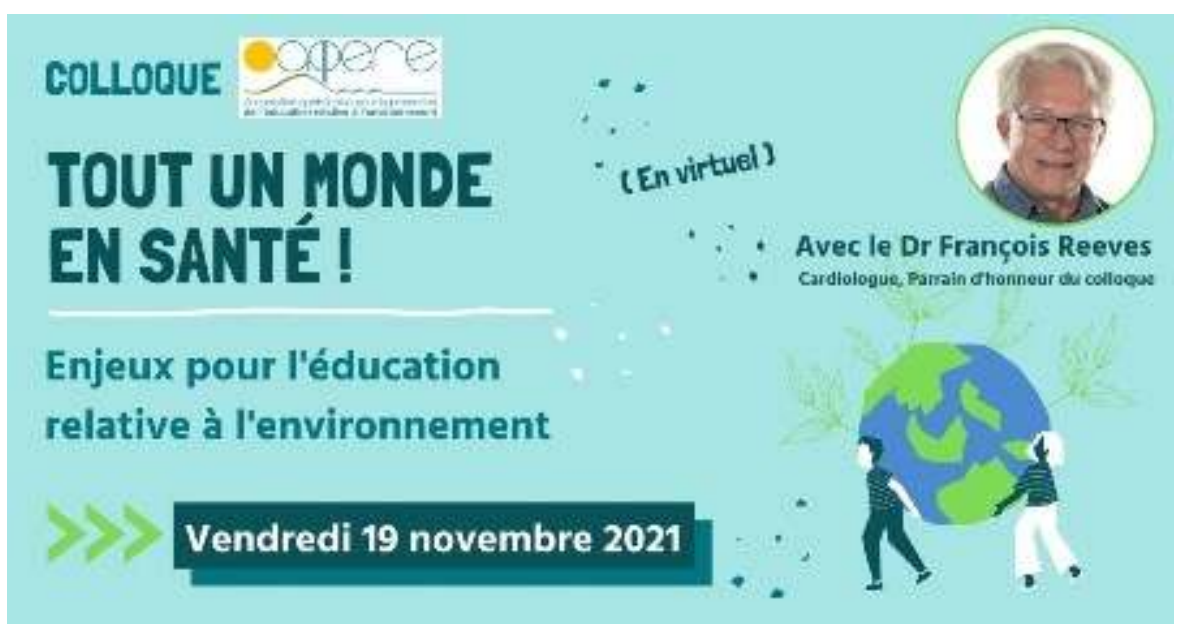

1 Les 19 novembre 2021 s'est tenu en mode virtuel le colloque « Tout un monde en santé ! Enjeux pour l'éducation relative à l'environnement ", organisé par l'Association québécoise pour la promotion de l'éducation relative à l'environnement (AQPERE) ${ }^{1}$.

2 D'entrée de jeu, Jacqueline Romano-Toramanian, présidente de l'AQPERE, présente la thématique :

La Covid 19 nous a fait cruellement prendre conscience que nous, êtres humains, faisons partie de quelque chose de plus grand que nous, le Vivant, au même titre que les animaux, les insectes, les plantes, les micro-organismes, tous les éléments 
de la biodiversité. Nous sommes toutes et tous inter-reliés, interdépendants dans un environnement sans frontières avec lequel nous ne faisons qu'un. Ce qui nous distingue en tant qu'humain c'est notre capacité de réfléchir et d'agir. Cela met en évidence notre responsabilité individuelle et collective de passer à l'action et de protéger par tous les moyens nos écosystèmes et notre biodiversité (...) Ce colloque met l'accent sur le lien entre la santé des écosystèmes et celle des humains. Réunir les forces vives des milieux de l'éducation formelle et non formelle, et celles des milieux de la santé permet de mieux traiter de santé environnementale en éducation relative à l'environnement.

3 Trois axes ont orienté les activités de l'événement, invitant les participants à traiter du rôle et des apports de l'éducation en matière de santé environnementale :

4 Axe 1 - Système politico-économique, santé humaine et santé des écosystèmes

- Nos modes de production et d'exploitation ;

- La transition énergétique

- L'engagement citoyen pour obtenir de meilleurs choix politico-économiques et la promotion de la santé environnementale.

5 Axe 2 - Choix individuels et collectifs : modes de vie et santé environnementale

- Les gestes/comportements/choix au quotidien ayant un impact sur la santé environnementale ;

- Les projets citoyens d'innovation écosociale en faveur de la santé environnementale ;

- La justice écologique et sanitaire au sein des milieux de vie.

6 Axe 3 - Corps, esprit et environnement sain

- La nature nourricière et guérisseuse ;

- Les rapports philosophiques avec la nature et le Vivant ;

- L'éducation dans et avec la nature ;

- Les Effets du contact avec la nature sur la santé physique et psychique ;

-L'écoanxiété : comment intervenir?

7 La présidente de l'AQPERE, Jacqueline Romano-Toramanian a ouvert le colloque en rappelant que le changement climatique et la perte de la biodiversité menacent la santé des humains et des écosystèmes. "Notre seul recours, c'est la mobilisation citoyenne. J'ajouterais que cela passe avant tout prioritairement par l'éducation et le soutien à l'engagement citoyen des jeunes et des moins jeunes. Dès lors, l'éducation relative à l'environnement et à l'écocitoyenneté ne peut plus être négligée par les instances politiques et reléguée à la marge des systèmes scolaires. »

8 Ensuite, Dr François Reeves, cardiologue d'intervention et parrain d'honneur de ce colloque, a mis en évidence que ce n'est pas l'apport scientifique seul qui a raison d'une crise, mais aussi l'attitude de la population. «De tout temps, une éducation de qualité permet de mieux cerner les enjeux, d'anticiper et d'opter pour les meilleures solutions. "

9 L'allocution d'Alain Deneault a ouvert à son tour une réflexion sur la façon d'aborder l'éducation relative à l'environnement et la santé environnementale: il importe d'équilibrer les sciences exactes et les Humanités en ouvrant notamment à la philo, aux arts et à l'esthétisme. Le professeur de philosophie a insisté également sur le fait que les bouleversements climatiques, la perte de biodiversité et autres problèmes socioécologiques associés nous obligent à repenser l'économie en fonction de la nature, du vivant, du territoire, du climat. 
10 l'environnement et à l'écocitoyenneté, a accueilli trois invités pour y partager leurs réflexions tant sur la thématique du colloque que sur le contenu des deux interventions précédentes. Lise Parent, écotoxicologue à l'université TELUQ, a présenté un important éclairage sur le concept de santé environnementale, invitant à cheminer dans un monde où les liens entre la santé humaine et celle de notre environnement demeurent difficiles à intégrer. Samuel Rainville, membre de la nation Innue de Pessamit et professionnel sur les dossiers autochtones à l'Université de Montréal, a ensuite traité de la valorisation des savoirs autochtones et du processus d'affirmation identitaire et culturelle chez les jeunes autochtones. Il a caractérisé la relation des populations autochtones à leur territoire de vie, ainsi que leurs représentations de la santé comme sources d'inspiration en matière de santé environnementale. Enfin, Nathalie Robitaille, inhalothérapeute, directrice de l'organisme Synergie Santé Environnement (SSE), s'est appuyée sur sa recherche portant sur l'éducation relative à la santé environnementale chez les jeunes, pour souligner l'importance de développer leur pouvoir-agir, essentiel à leur engagement.

11 Quant au panel de clôture, animé par Lucie Sauvé, professeure émérite de l'UQAM, il avait pour but de porter la voix des jeunes sur les deux questions suivantes, en les abordant dans une perspective de santé environnementale : d'abord, leur perception de la situation actuelle et leur vision d'avenir; et ensuite, ce qu'ils ont appris et ce qu'il faudrait apprendre. Trois intervenants ont ainsi partagé leurs regards, leurs craintes et leurs aspirations quant à l'évolution de notre monde: Albert Lalonde, étudiant en droit à l'UQAM, Marianne Renauld Robitaille, diplômée de la maîtrise en études politiques appliquées de l'Université de Sherbrooke, et Mélody Porlier, étudiante en médecine à l'Université de Montréal. Après avoir traité du rôle des jeunes en matière de santé environnementale, ils ont abordé la mission de l'école et des autres lieux d'éducation pour stimuler leur engagement.

Outre ces activités en plénière, plus de 30 ateliers ont été offerts dans le cadre du colloque. Ils ont permis d'aborder sous différents angles, les liens entre la santé humaine et celle des écosystèmes. Diverses propositions, initiatives, projets et ressources pédagogiques y ont été exposés et d'intéressantes discussions ont émergé des interactions entre les participants.

La mise en ligne de documents et de vidéos ${ }^{2}$, dont les allocutions d'introduction, les capsules vidéo de Riccardo Petrella, les panels d'ouverture et de clôture et plusieurs ateliers, invite à poursuivre l'auto-formation après l'événement et à soutenir la réflexion, la discussion et l'action pédagogique. Avec ce colloque, l'AQPERE poursuit sa mission de favoriser les échanges en éducation relative à l'environnement, de contribuer au partage de ressources pédagogiques et d'affiner les connaissances sur les enjeux environnementaux du Québec. 


\section{NOTES DE FIN}

1. https://www.aqpere.qc.ca

2. Les documents et enregistrements des contributions au colloque sont accessibles au lien suivant :

https://www.youtube.com/watch?

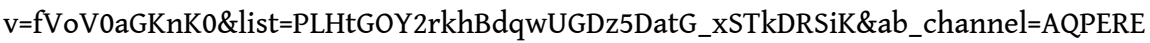

\section{AUTEUR}

\section{SANDRINE LOUAPRE}

Coordonnatrice de l'Association québécoise pour la promotion de l'éducation relative à

l'environnement (AQPERE) 